\title{
Tendencias y perspectivas del derecho cooperativo en el contexto global y la supervisión como oportunidad para el sector de la economía solidaria'
}

(Trends and prospects of cooperative law in the global context and the supervision as an opportunity for the solidarity economy sector)

Antonio Fici²

Universidad de Molise

Recibido: 05.05.2015

Aceptado: 17.07.2015

Sumario: I. Introducción. II. Tendencias del derecho cooperativo. 2.1. El papel del derecho cooperativo. 2.2. La identidad de las cooperativas. 2.3. La convergencia del derecho cooperativo. 2.4. La normalización de la sociedad cooperativa. 2.5. Privatización de funciones estatales y participación de organismos cooperativos. III. Funciones y modelos de supervisión cooperativa. IV. Bibliografía.

Resumen: Este artículo se divide en dos partes: en la primera se presentan y comentan las más recientes tendencias del derecho cooperativo en el contexto global; en la segunda, se examina la cuestión más específica de la supervisión de las cooperativas, siguiendo, también aquí, un enfoque comparativo. El hilo conductor es la identidad cooperativa. La tesis propuesta en el artículo es que la supervisión constituye un elemento indispensable de la legislación cooperativa en tanto en cuanto proteja de manera adecuada la específica identidad de las cooperativas, que la propia legislación cooperativa debería establecer, haciendo así frente al riesgo de la inoportuna asimilación de las cooperativas con las restantes sociedades, concretamente, las sociedades de capital con ánimo de lucro.

Palabras clave: Cooperativas, Derecho cooperativo, Derecho comparado, Supervisión cooperativa, Identidad cooperativa

Abstract: This article is divided into two parts: the first presents and discusses the most recent trends of cooperative law in the global context; the sec-

1 Ponencia presentada al «Congreso Sector Solidario. Motor para la prosperidad social y económica en Colombia», organizado por la Superintendencia de la Economía Solidaria de la República de Colombia (Bogotá, D. C., Colombia, 14 de octubre de 2014).

2 Correo electrónico: antonio.fici@unimol.it 
ond examines, also from a comparative legal perspective, the more specific issue of the supervision of cooperatives. The common thread is the cooperative identity. The article puts forward that cooperative supervision constitutes an essential element of cooperative legislation to the extent that it protects in an appropriate manner the specific identity of cooperatives, which the very cooperative legislation should establish in order to prevent cooperatives from being assimilated to other companies, notably, for-profit stock companies.

Key words: Cooperatives, Cooperative law, Comparative law, Cooperative supervision, Cooperative identity. 


\section{Introducción}

Este artículo se divide en dos partes: en la primera se presentan y comentan las más recientes tendencias del derecho cooperativo en el contexto global y que también expresan sus perspectivas de futuro; en la segunda, se examina la cuestión más específica de la supervisión de las cooperativas, siguiendo, también aquí, un enfoque comparativo. En realidad, las dos partes están sustancialmente interrelacionadas. El hilo conductor es la identidad cooperativa. La supervisión, como apuntaré más adelante, constituye un elemento indispensable de la legislación cooperativa en tanto en cuanto proteja de manera adecuada la específica identidad de las cooperativas, que la propia legislación cooperativa debería establecer, haciendo así frente al riesgo de la inoportuna asimilación de las cooperativas con las restantes sociedades, concretamente, las sociedades de capital con ánimo de lucro.

Debo admitir que tengo una especial obsesión por la cuestión de la identidad cooperativa y, más en general, de las entidades de la economía solidaria, porque estoy convencido de que preservar una identidad distinta - lo que sin embargo, como destacaré, no significa una identidad rígida e inmutable - resulta crucial para el desarrollo de la economía solidaria, frente a tentativas, cada vez más insidiosas, por parte del sector capitalista y de sus pensadores, de atenuarla, así que se puede decir que todas las entidades, todas las empresas, al final son iguales y, por lo tanto, merecen el mismo tratamiento jurídico para que no se viole el principio de competencia. Esto es lo que, en mi opinión, corremos el riesgo que suceda en Europa si algunas teorías norteamericanas sobre el concepto de empresa social se consolidan³.

Aquí, en Colombia y América Latina, probablemente este riesgo sea menor, puesto que hay oportunas disposiciones constitucionales que destacan la diferencia y la especial relevancia del sector de la economía solidaria (así como el compromiso de apoyo por parte del Estado) ${ }^{4}$. Sin embargo, aunque con diferente intensidad, el riesgo, a mi parecer, está presente en todas partes y es necesario que existan unas sólidas bases teóricas para hacerle frente. Sería preferible si hubiese un sustrato teórico común que fuera universalmente compartido entre todos aquellos

3 Véase $\mathrm{A}$. $\mathrm{FICl}$, «Función y modelos de regulación de la empresa social» (de próxima publicación).

${ }^{4}$ Cf., concretamente, artículos 38, 58, apartado 3, y 333, apartado 3, Constitución política de Colombia, 1991; y también C.L. FAJARDO-CALDERÓN, C.C. CABALCRUZ, O.A. DONNEYS-BELTRÁN, «La economía solidaria: de lo legal a la formación integral», en Criterio libre, número 9, 2008, p. 47 y ss. 
que creen que la economía solidaria contribuye a mejorar nuestra vida y el mundo en que vivimos, ya que la globalización precisa de instrumentos globales y los sujetos de la economía capitalista ya disponen de ellos.

\section{Tendencias del derecho cooperativo}

En 2013 se publicó una obra única en su género, una obra que tuve la oportunidad y el privilegio de coordinar con los ilustres colegas profesores Dante Cracogna y Hagen Henrÿ. Me refiero al International Handbook of Cooperative Law (o Manual Internacional de Derecho Cooperativo), publicado por la editorial alemana Springer ${ }^{5}$. Este volumen se divide en cuatro partes y se articula en 38 capítulos. Ofrece por vez primera y, además, en la misma lengua, el inglés, un panorama de más de 35 leyes cooperativas, más concretamente, 31 leyes nacionales y cuatro leyes supranacionales. Han contribuido a su redacción más de 30 autores de más de 30 países de todo el mundo. Además de una base conceptual común que se presenta en la primera parte, el Manual ofrece una oportunidad única de conocer las tendencias actuales del derecho cooperativo en el mundo, así como, lo que puede ser de gran ayuda para los legisladores, de conocer las normas vigentes en otros ordenamientos que puedan contribuir a resolver cuestiones concretas de regulación cooperativa en el propio país. En pocas palabras, el libro favorece la circulación a nivel global de disposiciones, ideas, y conceptos relativos al derecho cooperativo, lo que contribuye a edificar aquella base teórica común que, como mencionaba antes, es fundamental para el desarrollo de las cooperativas y de la economía social.

Mi opinión particular sobre las recientes tendencias y perspectivas futuras del derecho cooperativo, y que voy a presentarles a continuación, se basa principalmente en este volumen y en el trabajo que he realizado durante más de dos años como coordinador de esta obra.

Desde una perspectiva global, no limitada, entonces, a un particular ordenamiento jurídico, creo que es posible identificar cinco tendencias generales del derecho cooperativo, o más precisamente relativas al derecho cooperativo, que son:

1) una mayor conciencia del papel del derecho cooperativo en el éxito de las cooperativas y del movimiento cooperativo;

5 D. CRACOGNA, A. FICl, H. HENRY (eds.), International Handbook of Cooperative Law, Springer, Heidelberg, 2013. 
2) una orientación a adaptar por ley la identidad cooperativa a las exigencias concretas de las cooperativas;

3) una tendencia a la convergencia de los derechos cooperativos nacionales;

4) una inclinación hacia la normalización de la sociedad cooperativa frente al enfoque más ideológico que caracterizó tiempos menos recientes;

5) la privatización de funciones estatales relativas a las cooperativas y participación de los organismos representativos del movimiento cooperativo en el desarrollo de las mismas.

Es verdad que estas tendencias, como veremos a continuación, están relacionadas entre sí, porque todas son manifestaciones, o por lo menos eso creo yo, de un nuevo período para el derecho cooperativo y la ciencia jurídica cooperativa. Por ejemplo, como veremos, la cuestión de la identidad vuelve a ser central en tanto en cuanto crece la conciencia del papel central de la ley para el desarrollo de las cooperativas. Al mismo tiempo, el proceso de normalización no puede sino influir en el contenido de esta identidad. Por otro lado, la privatización de las funciones estatales relativas a las cooperativas se desarrolla paralelamente al proceso de normalización de la cooperativa, cada vez más entendida como una forma jurídica de ejercicio de empresa entre las otras formas jurídicas: no ya la mejor, sino una específica forma, diferente de todas las demás.

Además, debo añadir que las tendencias que acabo de mencionar afectan también a la cuestión de la supervisión, como veremos después.

¿Hacia dónde va el derecho cooperativo, entonces?

\subsection{El papel del derecho cooperativo}

Como decía, la primera tendencia que podemos identificar es la creciente difusión entre los pensadores y los líderes del movimiento cooperativo de la idea de que el derecho cooperativo es de especial importancia para la defensa y el desarrollo de las cooperativas y del cooperativismo. Esto era algo que ya estaba subrayado en las Directrices de las Naciones Unidas de 2001 para el desarrollo de las cooperativas ${ }^{6}$ y en la Recomendación 193 de la Organización Internacional del Tra-

${ }^{6}$ Cf. Resolución n. ${ }^{\circ}$ 56/114 de las Naciones Unidas, aprobada en la 88. ${ }^{a}$ sesión plenaria de la Asamblea General de 19 de diciembre de 2001. 
bajo (OIT) de 2002 sobre la promoción de las cooperativas7, así como en la Comunicación de 2004 de la Comisión europea sobre la promoción de las cooperativas en Europa ${ }^{8}$. Sin embargo, sólo en los últimos años parece que se haya tomado verdaderamente en serio, sobre todo por parte de los organismos de representación del movimiento cooperativo internacional.

Hay una serie de circunstancias que lo demuestran:

- en el Plan de la Alianza Cooperativa Internacional (ACI) para una década cooperativa de enero $2013^{9}$ se afirma de forma general que: «el marco jurídico desempeña un papel crítico en la viabilidad y la existencia de las cooperativas», y hay un capítulo específico sobre los marcos jurídicos, en donde se establece el objetivo de "garantizar marcos jurídicos que apoyen el crecimiento cooperativo», también identificando, entre las acciones posibles para lograrlo, el estudio comparativo del modo en el que las leyes se aplican a las cooperativas, y en particular indicando, como una ayuda posible en este sentido, la iniciativa del Grupo de Estudio sobre el Derecho Cooperativo Europeo (SGECOL), el Grupo basado en Trento que tengo el privilegio de coordinar y al que me referiré más adelante;

- posteriormente, la propia $\mathrm{ACl}$ instituye en su seno un nuevo comité temático, el Comité de Derecho cooperativo, que se compone de dos expertos jurídicos por cada región de la $\mathrm{ACl}$ (yo mismo participo en este comité como experto por Europa) y de un Presidente (que actualmente es mi colega Hagen Henrÿ). Entre sus tareas institucionales se encuentran la de proporcionar a la $\mathrm{ACl}$ consultas independientes sobre todas las normas que dan forma a la entidad cooperativa y regulan sus operaciones; formular propuestas para convertir los principios legales en derecho cooperativo a fin de garantizar la identidad cooperativa; y la de evaluar, aconsejar, proponer y monitorizar los cambios de política cooperativa a nivel nacional, regional, internacional y global que puedan afectar al derecho cooperativo ${ }^{10}$;

7 Cf. Recomendación n. 193/2002 de 20 de junio de 2002 de la Organización Internacional del Trabajo (OIT).

8 Cf. Comisión Europea, Comunicación sobre el fomento de las sociedades cooperativas en Europa, COM (2004) 18 final, de 23 de febrero de 2004.

9 Cf. Alianza Cooperativa Internacional, Plan para una década cooperativa, enero 2013, en http://www.aciamericas.coop/IMG/pdf/ica_blueprint_es.pdf.

10 Véase http://ica.coop/en/alliances-committees-and-networks. 
- el derecho cooperativo aparece cada vez más en los congresos y cumbres internacionales de cooperativas. Ya en 2012, que fue un año particular para las cooperativas ${ }^{11}$, hubo una pequeña sección de derecho cooperativo en el congreso de Manchester de la $\mathrm{ACl}$, que fue organizado por el propio grupo SGECOL ${ }^{12}$. También se centró especialmente en el derecho cooperativo la Conferencia regional de la ACl Américas de octubre 2013 en Guarujá, Brasil, donde tuvo lugar un congreso continental de derecho cooperativo organizado por el profesor Dante Cracogna de Argentina, y en el que participó un gran número de juristas cooperativos de la región y no sólo ${ }^{13}$. Y finalmente en la tercera Cumbre Cooperativa de las Américas de noviembre 2014 en Cartagena, Colombia, habrá un foro acerca de la actualización en derecho cooperativo continental ${ }^{14}$;

- los proyectos sobre el derecho cooperativo comparado están financiados con mayor frecuencia cada vez por cooperativas y encuentran el favor de los investigadores, que gracias a este impulso constituyen grupos transfronterizos de investigación. Un ejemplo de ello es el grupo SGECOL, que ya hemos visto mencionado por la $\mathrm{ACl}$ en su plan para una década cooperativa. Cabe ahora reseñar brevemente su estructura, objetivos y metodología porque dan forma a un modelo que está alcanzando una popularidad cada vez mayor (hay quien está pensando en constituir grupos parecidos en otros continentes) y que puede ser de gran utilidad en todo continente, y no solo en Europa, para abordar cuestiones concretas de legislación cooperativa ${ }^{15}$.

El Grupo de Estudio sobre el Derecho Cooperativo Europeo (o SGECOL por sus siglas en inglés) es un grupo de estudiosos del derecho

11 Las Naciones Unidas lo proclamaron año internacional de las cooperativas.

12 El congreso "Co-operatives United» tuvo lugar en Manchester, Reino Unido, 29 octubre-2 noviembre de 2012. La sección sobre el derecho cooperativo fue organizada por el professor lan Snaith, que es también miembro del grupo SGECOL.

13 Véase ahora Congreso Continental de Derecho Cooperativo (Guarujá, San Pablo, Brazil, 8, 9 y 10 de octubre de 2013), Buenos Aires, Intercoop, 2014.

14 Esta cumbre tuvo lugar en Cartagena de India, Colombia, 2-7 noviembre, 2014: véase $\mathrm{http}: / / \mathrm{ww}$.aciamericas.coop/Invitacion-5384.

15 Para saber más, véase G. FAJARDO, A. FICI, H. HENRŸ, D. HIEZ, D. MEIRA, H.-H. MÜNKNER, y I. SNAITH, «El Nuevo grupo de estudio en Derecho cooperativo europeo y el proyecto "Ios principios del Derecho cooperativo europeo"», en CIRIEC-España. Revista Jurídica de Economía Social y Cooperativa, número 24, p. 331 y ss.; y la página web del grupo: http://www.euricse.eu/it/gruppo-di-studio-sul-diritto-europeo-delle-societa-cooperative/. 
cooperativo de distintos países europeos (once países están actualmente representados), que se reunieron por primera vez en Trento en noviembre de 2011 y siguen reuniéndose periódicamente gracias al apoyo financiero de EURICSE, European Research Institute on Cooperative and Social Enterprises, que a su vez es un instituto financiado por la provincia de Trento y el movimiento cooperativo de esta provincia italiana.

SGECOL tiene como objetivo general la investigación comparada en derecho cooperativo para promover un mayor conocimiento y comprensión de esta rama del derecho entre los juristas, académicos y los gobiernos a nivel nacional, europeo e internacional. SGECOL persigue sus objetivos a través de distintos proyectos relacionados con el derecho cooperativo, empezando por la redacción de los Principios de Derecho Cooperativo Europeo (PECOL).

LoS PECOL son principios «ideales» de derecho cooperativo que tendrán la forma de disposiciones normativas acompañadas de comentarios explicativos (que los autores consideran particularmente importantes desde una perspectiva de comparación jurídica), y se desarrollarán a partir del derecho cooperativo existente en Europa, concentrándose en particular en siete países (Alemania, España, Finlandia, Francia, Italia, Portugal y Reino Unido).

Sin embargo, el proyecto de los PECOL no se limitará a resumir y describir el núcleo compartido de la legislación nacional. El proyecto irá más allá para presentar las principales disposiciones generales a través de las cuales -en la opinión de los autores- se debería formular la legislación sobre cooperativas, de modo que, dote a las cooperativas de una identidad jurídica firme y distinta de la de otras formas de organización empresarial. Los PECOL, entonces, se concentrarán en la identidad de las cooperativas y, en este respecto, serán más detallados que los principios de la $\mathrm{ACl}$. Estos últimos principios, aunque de hecho contribuyen a dar forma a la identidad cooperativa, son a veces demasiado generales y carentes de algunas precisiones que serían esenciales para este fin. LOS PECOL, en cambio, pretenden hacer de intermediarios entre los principios de la $\mathrm{ACl}$ y la propia legislación cooperativa ${ }^{16}$.

En definitiva, los hechos que acabo de mencionar demuestran, indudablemente, que el derecho cooperativo ha suscitado una gran atención, lo que, debo decir, no me sorprende, sino todo lo contrario, me parece una tendencia que debería haberse manifestado mucho antes,

16 Un borrador de los PECOL se presentará en un seminario del 9 de junio de 2015 en Bruselas. 
puesto que, en mi opinión, no se puede separar el destino de la cooperativa como forma distinta de empresa de un marco jurídico que reconozca y preserve su peculiar identidad. Sólo gracias a la ley, y no lo digo porque sea jurista, es posible ofrecer la prueba inequívoca del hecho que las cooperativas son diferentes de otras entidades empresariales, sobre todo frente a los que mantienen lo contrario (y que muchas veces, es superfluo decirlo, son capitalistas). Permítanme que les ofrezca un claro ejemplo de esto.

Debido a la gran relevancia que en los Tratados de la Unión europea asume el principio de la libre competencia entre las empresas en el mercado único europeo y a la específica prohibición de las ayudas de Estado a las empresas que puedan perjudicar a la competencia, en la Unión Europea se ha discutido mucho acerca de la legitimidad del tratamiento fiscal que algunos países miembros de la Unión otorgan a las cooperativas, puesto que este tratamiento es más favorable que el otorgado a las empresas que no tienen una forma cooperativa. Para los organismos representativos del movimiento cooperativo europeo ha sido muy difícil demostrar que las cooperativas son diferentes de las organizaciones empresariales ordinarias $\mathrm{y}$, por lo tanto, merecen un tratamiento que no es más favorable sino específico de éstas, antes de que, en 2003, se aprobase el Reglamento de la Unión europea sobre la sociedad cooperativa europea ${ }^{17}$. Volveré más tarde sobre este reglamento, cuando me refiera a la tendencia a la convergencia del derecho cooperativo. Por ahora sólo quiero señalar que este mismo Reglamento asumió un papel decisivo para que, en 2011, el Tribunal de la Unión declarase potencialmente compatible con el derecho comunitario el tratamiento fiscal (que en aquel caso era italiano) de las cooperativas ${ }^{18}$.

17 Para un análisis detallado de este Reglamento europeo, véase A. FICl, «La Sociedad Cooperativa Europea: cuestiones y perspectivas», en CIRIEC-España. Revista Jurídica de Economía Social y Cooperativa, número 25, 2014, p. 69 y ss.

18 Cf. Tribunal de Justicia de la Unión Europea, 8 de Septiembre de 2011 (C-78/08 a C-80/08). La sentencia del TJUE tiene origen en una petición de decisión prejudicial planteada por la Corte Suprema Italiana (Corte Suprema di Cassazione) según lo establecido en el art. 267 del Tratado de Funcionamiento de la Unión Europea (TFUE) (antiguo art. 234 del Tratado constitutivo de la Comunidad Europea - TCE), y relativa a la compatibilidad con el Derecho comunitario de las exenciones fiscales otorgadas por la ley italiana a las sociedades cooperativas de producción y trabajo. Más concretamente, la Corte Suprema Italiana planteó sustancialmente la cuestión acerca de si estas ventajas podrían considerarse ayuda de Estado y, de ser así, en qué medida en la acepción del art. 107 del TFUE (antiguo art. 87 TCE). Las medidas fiscales estaban previstas en el art. 11, Decreto Presidencial n. ${ }^{\circ}$ 601/1973. Para un comentario, véanse A. FICl, «La Sociedad Cooperativa Europea: cuestiones y perspectivas», cit., y G. FAJARDO, "La especificidad de las sociedades cooperativas frente a las sociedades mercantiles y la legiti- 
El Tribunal llegó a la conclusión de que las cooperativas se encuentran en una situación de hecho y de derecho no comparable a las de las sociedades comerciales, lo que justifica su tratamiento fiscal diferente, y sacó esta distinta identidad del propio Reglamento de la SCE. De no haber sido así, por lo tanto, esta conclusión, de gran relevancia para el desarrollo de las cooperativas, no habría sido cierta. Quiero señalar que esta discusión es importante no sólo para las cooperativas, sino también para todas las empresas sociales o entidades de la economía social o solidaria.

\subsection{La identidad de las cooperativas}

Al examinar la primera tendencia, ya hemos hecho referencia a la identidad cooperativa como tema central del debate sobre el derecho cooperativo y núcleo fundamental de la legislación cooperativa.

En efecto, el papel de la ley en el desarrollo de las cooperativas se centra principalmente en establecer y preservar su identidad distinta ${ }^{19}$.

No obstante, si por un lado la ley debe asegurar a las cooperativas una identidad específica, por otro lado, la ley también puede concurrir a edificar esta identidad. A este respecto, surge la que, a mi entender, constituye la segunda tendencia del derecho cooperativo a nivel global, concretamente, la tendencia por parte de los legisladores a interpretar y relajar la identidad cooperativa, así como la establecida por la $\mathrm{ACl}$ en sus principios, para que las cooperativas sean dotadas de herramientas para su buen gobierno y finanzas.

Debo precisar que algunos estudiosos ya han hecho referencia a esta tendencia aunque, en términos negativos, hablando por ejemplo de la "societarización» de la cooperativa como resultado de una regulación cada vez más parecida a la de una sociedad comercial, lo que también ha conducido a otros a hablar de «isomorfismo», en la medida en que todas las formas jurídicas resultan iguales en virtud de este proceso. Más concretamente, según el profesor Henrÿ, la evolución del derecho cooperativo puede dividirse en dos fases: la primera, marcada por la distinción entre las cooperativas y las sociedades de capital, mientras que la segunda, que comienza en los años setenta del siglo pasado, se caracteriza por la aproximación entre unas y otras, es

midad de su particular régimen jurídico y fiscal según el tribunal de justicia de la Unión Europea», en Revista de derecho mercantil, 2013, p. 189 y ss.

19 Cf. A. FICl, «The Essential Role of Cooperative Law», en Dovenschmidt Quarterly, 2014 (de próxima publicación). 
decir, por la alineación de las cooperativas con las sociedades comerciales ${ }^{20}$.

Sin embargo, yo creo que esta tendencia no puede ser evaluada de forma tan negativa si, y en la medida en que, la ley continúa proporcionando una identidad distinta de las cooperativas, aunque dicha identidad sea o pueda parecer más relajada de lo que lo era antes o de lo que se entiende de una estricta lectura de los principios de la $\mathrm{ACl}$.

Ninguna identidad es, de hecho, inmutable, y una identidad rígida puede crear una separación perjudicial para los propios sujetos a cuya identidad nos referimos, en este caso las cooperativas. Como ha subrayado el profesor Dante Cracogna: «las circunstancias económicas del mundo actual plantean perentorias exigencias a las que deben dar respuesta estas entidades y para lo cual el marco jurídico debe realizar las adecuaciones necesarias. Tales adecuaciones entrañan un desafío a la vigencia de los principios que tradicionalmente informan las legislaciones de la materia» ${ }^{21}$. Además, si se añade el hecho de que los movimientos cooperativos nacionales están normalmente implicados en los procesos de cambios legislativos relativos a las cooperativas, se puede concluir que una cierta flexibilidad de la identidad cooperativa no sólo no es perjudicial, sino que además es requerida por las propias cooperativas.

Por supuesto, la posibilidad de adaptar la identidad cooperativa conlleva la cuestión de establecer qué adaptaciones son compatibles con la naturaleza específica de las cooperativas y cuáles son, en cambio, las que alteran esta naturaleza de manera inaceptable. Esto puede implicar, además, distinguir entre diferentes tipologías de cooperativas.

Me limito aquí a dos ejemplos.

Primero. El principio «un socio, un voto» no parece sufrir excepciones en la declaración de identidad cooperativa de la $\mathrm{ACl}$, por lo menos en lo que se refiere a las cooperativas de base o de primer grado 22 . Sin embargo, algunas leyes cooperativas (y no sólo en Europa) permiten a los estatutos sociales desviarse de esta regla previendo el voto plural (mientras que otras, por supuesto, como la Colombiana de 1988, no lo

20 Cf. H. HENRŸ, Orientaciones para la legislación cooperativa, Segunda edición, Organización Internacional del Trabajo, Ginebra, 2013, p. 9-10.

21 D. CRACOGNA, «Problemas actuales del derecho cooperativo», en Revista de derecho privado y comunitario, número 3, 2011, p. 12.

22 Segundo principio $\mathrm{ACl}$ : «En las cooperativas de base los miembros tienen igual derecho de voto (un miembro, un voto), mientras en las cooperativas de otros niveles también se organizan con procedimientos democráticos». 
permiten) ${ }^{23}$ ¿Cuándo deben considerarse legítimas estas excepciones? Los que critican la «societarización» de la forma cooperativa no pueden sino concluir que la excepción nunca sería justificable. Al contrario, yo pienso que no hay una respuesta única y que depende del criterio particular en el que se basa la excepción y en el tipo de cooperativa a la que se aplica. Por ejemplo, considero oportuno admitir por ley la posibilidad de voto plural ponderado en cooperativas de empresarios si el voto ponderado se asigna en proporción a la cantidad de actividad cooperativizada realizada (o de actos cooperativos realizados) por cada socio con la cooperativa. Esta previsión estatutaria posibilitaría que se hiciera frente a situaciones de heterogeneidad que no permitirían a la cooperativa actuar bien o incluso constituirse. Esto puede conllevar el éxito de la cooperativa o el uso de esta forma jurídica hasta en presencia de circunstancias desfavorables.

Segundo. Los principios de la ACI parecen aunar en la figura de socio de cooperativa tanto la calidad de usuario (o trabajador) de la empresa cooperativa como la calidad de aportador de su capital, de manera que en una cooperativa no podrían configurarse ni socios no usuarios ni usuarios no socios 24 .

En realidad, en las leyes cooperativas (y no solo europeas) hay una tendencia a permitir que las cooperativas admitan socios no usuarios como los socio inversores, que participan no ya para realizar actos cooperativos con la cooperativa sino solamente para invertir sus capitales (cualquiera que sea la razón específica para ello); y también hay una tendencia a permitir a las cooperativas realizar actividad con terceros no socios de la misma naturaleza que la que realizan con sus socios ${ }^{25}$.

Aquí se plantea nuevamente la misma cuestión: ¿Estamos ante excepciones compatibles o incompatibles con la identidad de la cooperativa? Si nos centramos en los principios de la $\mathrm{ACl}$ y los tomamos literalmente, las excepciones serían siempre incompatibles. De ahí nuevamente la idea de la «societarización» como una tendencia negativa del derecho cooperativo contemporáneo. Si, por el contrario, nos centramos en las posibles ventajas de estas medidas aparentemente poco ortodoxas, entonces podemos concluir que, en algunos casos, la no

23 Véase el art. 33, inciso primero, de la ley colombiana n. ${ }^{\circ} 79$ de 1988, por la cual se actualiza la legislación cooperativa; y para más referencias legislativas, cf. A. FICl, "Cooperative Identity and the Law», en European Business Law Review, 2013, p. 37 y ss.; A. FICl, «An introduction to cooperative law», en D. CRACOGNA, A. FICl, H. HENRY (eds.), International Handbook of Cooperative Law, cit., p. 3 y ss.

${ }^{24} \mathrm{Cf}$. sobre todo el primer y el tercer principio de la $\mathrm{ACl}$.

25 Referencias legislativas pueden encontrarse en $\mathrm{A}$. $\mathrm{FICl}$, «Cooperative Identity and the Law», cit.; y A. FICl, «An introduction to cooperative law», cit. 
coincidencia de socio y usuario de la empresa cooperativa es compatible con la identidad cooperativa. Por ejemplo, no tiene sentido limitar la actividad de la cooperativa con terceros no socios cuando los terceros, aunque invitados, no quieran hacerse socios de la cooperativa (pensamos en una cooperativa grande de consumo) o cuando los resultados de la actividad con terceros no socios se destinen a la educación y promoción cooperativa, o aún cuando la cooperativa actúa, no ya en el interés exclusivo de sus socios sino también en el interés de terceros o de la comunidad (pensamos en una cooperativa de solidaridad social o de interés general). Asimismo, los socios inversores no constituyen un asunto problemático cuando tienen un peso limitado en el gobierno de la cooperativa, o cuando los socios inversores son organismos de fomento del cooperativismo.

En conclusión, revisar y relajar la identidad cooperativa no siempre es una tendencia negativa del derecho cooperativo, pudiendo esta tendencia contribuir al desarrollo ulterior del modelo cooperativo de empresa. De nuevo, cabe subrayar que esta conclusión es válida también con referencia a otras entidades de la economía social o solidaria. Definir la identidad es un proceso que conlleva costes y beneficios que necesitan una atenta evaluación. Una identidad inmutable y granítica no siempre es positiva. Un desafío para el derecho cooperativo, así como para el derecho de las entidades de la economía solidaria, es adaptarse y articularse sin perder su propia identidad.

\subsection{La convergencia del derecho cooperativo}

Las cooperativas se inspiran en principios comunes de alcance universal. Estos principios derivan de la experiencia de la cooperativa de Rochdale, que tuvo el gran mérito de codificarlos, y han sido transmitidos hasta nuestros días gracias a las propias cooperativas y a sus organismos de representación como la $\mathrm{ACl}$. Por tanto, si por un lado es verdad lo que afirma el profesor Henrÿ, es decir, que en el derecho cooperativo se reflejan las circunstancias económicas, sociales y políticas da cada país, por otro lado, sin embargo, una mayor uniformidad entre los derechos cooperativos nacionales, por lo menos en lo que se refiere a los elementos de la identidad cooperativa, no sólo iría de acuerdo con la aspiración a la universalidad que siempre ha caracterizado al movimiento cooperativo, sino que sería también ventajosa para las cooperativas. De hecho, una identidad más uniforme en tiempos de globalización contribuiría al desarrollo de la forma cooperativa de empresa, porque permitiría expresar con mayor facilidad la peculiar identidad 
cooperativa frente a actores internacionales o supranacionales, que determinan cada vez más el destino del mundo. Ya he hecho referencia a la cuestión de la tributación cooperativa en Europa. En este caso, el reglamento europeo sobre la SCE, entonces una ley supranacional, fue decisivo para que un organismo supranacional como el Tribunal de la Unión Europea emitiera aquella decisión que fue muy favorable para las cooperativas constituidas con arreglo al derecho nacional de un Estado miembro de la Unión. Además, hay que añadir que la mayor uniformidad legislativa favorece la actividad transfronteriza de las cooperativas, lo que constituye también un factor de desarrollo legislativo, debido al fenómeno de la posible competencia entre ordenamientos jurídicos.

Por tanto, una tendencia que me parece positiva del derecho cooperativo contemporáneo es la que va hacia la convergencia de los derechos cooperativos nacionales. Este proceso está favorecido por diversos instrumentos:

- instrumentos de uniformización legislativa en sentido estricto como, por ejemplo, la regulación uniforme de cooperativas que fue aprobada en 2010 por la Organización para la Armonización de la Legislación Empresarial en África (OHADA) y se aplica en 17 Estados de África, que como consecuencia tienen exactamente la misma ley cooperativa26;

- instrumentos que favorecen la aproximación legislativa ofreciendo un modelo de ley cooperativa que los legisladores nacionales pueden utilizar como, por ejemplo, la Ley Marco para las cooperativas de América Latina de 200827;

- instrumentos que proporcionan una normativa supranacional de cooperativas que los legisladores nacionales pueden tomar como modelo para adaptar sus leyes nacionales y, en algunos casos, deben hacerlo así si quieren evitar que las cooperativas se constituyan con arreglo al derecho supranacional en lugar de con arreglo al derecho nacional. Estos instrumentos, entonces, no ayudan directamente a la armonización de las legislaciones nacionales, sino que la promueven de forma indirecta, es decir, de facto. Éste es el caso del reglamento de la Unión Europea sobre la sociedad cooperativa europea (SCE), que ya hemos mencio-

26 Véase D. HIEZ y W. TADJUDJE, «The OHADA Cooperative Regulation», en D. CRACOGNA, A. FICI, H. HENRY (eds.), International Handbook of Cooperative Law, cit., p. 89 y ss.

27 Alianza Cooperativa Internacional para las Américas, Ley marco para las cooperativas de América Latina, San José, Costa Rica, 2009. 
nado. Este reglamento ofrece un modelo de cooperativa alternativo al ofrecido por los ordenamientos nacionales, de modo que los ciudadanos y las entidades jurídicas de la Unión puedan decidir constituir una sociedad cooperativa europea en lugar de una sociedad cooperativa nacional. Evidentemente, el reglamento de la SCE es un instrumento legislativo supranacional y no de uniformización o armonización de las legislaciones nacionales, pero puede tener, y de hecho ha tenido en parte, efectos indirectos de aproximación de las leyes cooperativas nacionales;

- la tendencia a la convergencia se encuentra también favorecida por estudios de derecho cooperativo comparado y por iniciativas como la constitución de equipos o comités de investigadores de diversos países que, como ya hemos visto, son hechos de gran actualidad.

\subsection{La normalización de la sociedad cooperativa}

La cuarta tendencia, la de la normalización de la cooperativa, se percibe más con referencia a la doctrina jurídica que al derecho cooperativo en sentido estricto, donde sin embargo, no faltan manifestaciones de este fenómeno. Es la tendencia a ver, analizar y regular la cooperativa cada vez de forma más sencilla como un tipo jurídico de empresa entre otros tipos jurídicos que se pueden elegir a la hora de decidir cómo desarrollar una determinada actividad económica. Como se subrayaba, entonces, no ya la mejor forma jurídica por principio, sino una forma específica, diferente de todas las demás. Algunos índices de esta tendencia son:

- el desarrollo de estudios comparativos entre cooperativas y otras formas jurídicas de empresas, incluso las sociedades comerciales, dirigidos a realizar un análisis de costo-beneficio comparativo de estos tipos diferentes de organizaciones, que, entre otras cosas, pueda servir como orientación para la elección de la forma jurídica más adecuada para un determinado tipo de actividad económica (los estudios del profesor Henry Hansmann son ejemplos en este sentido) ${ }^{28}$;

${ }^{28} \mathrm{Cf}$. H. HANSMANN, The ownership of enterprise, HUP, Cambridge-London, 1996. 
- a nivel legislativo, por ejemplo, un índice es la previsión de la posibilidad de que la cooperativa esté formada también por entidades no cooperativas, incluidas sociedades comerciales, que quieran colaborar entre sí utilizando esta forma en lugar de otras que para estas últimas son más tradicionales (como, por ejemplo, el grupo de sociedades). Otro ejemplo es la reducción del número mínimo de socios para la constitución de una cooperativa, que ahora es tres por término medio.

Este enfoque se diferencia claramente del enfoque puramente ideológico que ha caracterizado, y en algunos lugares todavía caracteriza, la visión del fenómeno cooperativo. Personalmente, creo que un enfoque puramente ideológico de las cooperativas, aunque necesario en el momento en que surge este tipo particular de organización empresarial, puede tener, sin embargo, un efecto perjudicial para su crecimiento ulterior una vez que la cooperativa se ha consolidado y es reconocida como una forma diferente y alternativa de realizar negocios. Cuando sea este el caso, la normalización puede beneficiar a las cooperativas, como veremos al hablar de la quinta tendencia. Además, la normalización de la forma jurídica cooperativa es consistente con la opinión de que las cooperativas son parte esencial, pero no única, de un modelo pluralista de mercado integrado por diferentes actores con motivaciones diversas, que académicos destacados (como Joseph Stiglitz) consideran algo especialmente beneficioso a nivel macroeconómico 29 .

Volviendo a la ciencia jurídica, creo que las cooperativas no deben ser aisladas de las demás organizaciones empresariales. Deben ser tratadas como una forma jurídica más entre las diferentes formas jurídicas existentes. Ha de tenerse en cuenta el riesgo del «alineamiento» de la forma jurídica cooperativa con las sociedades comerciales, pero ello no implica que el estudio de las cooperativas deba realizarse de forma independiente del estudio de las sociedades comerciales y de otras organizaciones empresariales. Siempre y cuando la identidad cooperativa sea preservada, el derecho de las sociedades y, más en general, el derecho comercial, pueden contener disposiciones que sean útiles para la regulación de las cooperativas. Esto es particularmente cierto en aquellos países que realizan mayores inversiones para la mejora del derecho de las sociedades, o en los que la teoría jurídica cooperativa está prácticamente ausente.

29 Cf. J. STIGLITZ, "Moving beyond market fundamentalism to a more balanced economy», en Annals of Public and Cooperative Economy, 2009, p. 348 y ss. 
Además, los juristas especializados en cooperativas no deberían realizar sus estudios de manera independiente de los juristas especializados en otras ramas del derecho. Para que la teoría jurídica cooperativa prospere y para incrementar la visibilidad de las cooperativas, necesitamos más artículos sobre derecho cooperativo en revistas de derecho de sociedades y de derecho comercial; necesitamos que se imparta el derecho cooperativo dentro de (o en relación con) los cursos sobre derecho de sociedades o derecho comercial. La idea de la especificidad del derecho cooperativo no puede conducir a la marginalización de esta disciplina y de quienes la practican. Aprendemos mucho más sobre las cooperativas comparándolas con otras formas de empresa. Y de ese modo, nosotros, como juristas especializados en la materia, incrementamos la visibilidad de las cooperativas.

\subsection{Privatización de funciones estatales y participación de organismos cooperativos}

La quinta tendencia que voy a presentar está relacionada con las precedentes $y$, en particular, con la cuarta, referente al tema de la supervisión que abordaré en la segunda parte de esta ponencia, y se refiere a un aspecto muy sensible de la relación entre el Estado y las cooperativas.

Cada vez con más frecuencia, las leyes cooperativas transfieren o atribuyen a entidades cooperativas o a organismos integrados por representantes del movimiento cooperativo funciones relativas a cooperativas que tradicionalmente eran ejercidas por el Estado.

Hay muchos ejemplos de esto.

En la provincia de Quebec, en Canadá, el "Conseil de la coopération du Québec», un organismo integrado por federaciones de cooperativas, ejerce por delegación de ley o acuerdos con la autoridad pública, una serie de funciones, como el control sobre el registro de nuevas entidades cooperativas, la recogida de datos estadísticos sobre las cooperativas y la implementación de programas de desarrollo cooperativo ${ }^{30}$.

En Alemania, la revisión previa al registro de nuevas cooperativas está atribuida a federaciones de cooperativas ${ }^{31}$.

30 Cf. T. PETROU, «Canada», en D. CRACOGNA, A. FICI, H. HENRY (eds.), International Handbook of Cooperative Law, cit., p. 289 y ss.

31 Cf. H.-H. MÜNKNER, «Germany», en D. CRACOGNA, A. FICl, H. HENRY (eds.), International Handbook of Cooperative Law, cit., p. 413 y ss. 
Otro ejemplo lo tenemos también en la ley cooperativa colombiana de 1988, donde se prevé que los recursos financieros públicos para el desarrollo y fomento cooperativos sean canalizados preferentemente a través de los organismos cooperativos de segundo grado e instituciones auxiliares de cooperativismo de carácter financiero (art. 134), organismos que las cooperativas podrán organizar según lo previsto por los artículos 98 y 99 de la misma ley (aunque, en la práctica, el Estado ha empleado esta medida sólo al principio).

Ante todo, cabe subrayar que esta tendencia se refiere también a la función de supervisión cooperativa. En algunos ordenamientos como Alemania e Italia, las federaciones (o asociaciones o uniones) de cooperativas ejercen la supervisión sobre las cooperativas, mientras que en otros ordenamientos se prevé la formación de organismos específicamente dedicados a la supervisión y desarrollo de las cooperativas en los que se involucra a las organizaciones representativas del movimiento cooperativo ${ }^{32}$.

A mi parecer, esta tendencia es muy positiva para las cooperativas porque fortalece, en una perspectiva de normalización de la forma cooperativa, la autonomía e independencia de las cooperativas, según lo previsto también por la $\mathrm{ACl}$ en sus principios ${ }^{33}$. De todas formas, medidas como las mencionadas funcionan siempre y cuando haya un movimiento cooperativo lo suficientemente consolidado como para desempeñar funciones de autogobierno, porque, si no es éste el caso, el papel del Estado permanece irrenunciable. Esto es algo que debe tenerse en cuenta también a la hora de analizar el tema de la supervisión y de sus posibles modalidades de implementación. Lo que nos conduce a la segunda y última parte de la ponencia, sobre la función y los modelos de supervisión cooperativa.

\section{Funciones y modelos de supervisión cooperativa}

Las cooperativas constituyen el único tipo de organización empresarial que se encuentra sujeto a una forma de control externo dirigido a verificar el cumplimiento de las normas que definen y regulan su objetivo y estructura $y$, por lo tanto, el fin mutualista, la democracia interna, la participación de los socios, etc. Normalmente, en organizaciones de otro tipo, si se exceptúan las sociedades cotizadas, este control

32 Cf. infra en el texto.

33 Véase en particular el cuarto principio de la $\mathrm{ACl}$ sobre la autonomía e independencia de las cooperativas. 
corresponde a los propios socios, es decir, es solamente interno. En las cooperativas, en cambio, este control es también externo y muy peculiar, y suele adoptar el nombre de "supervisión cooperativa», aunque también se pueden encontrar en la ley otros términos como «vigilancia» o «fiscalización». La función de control normalmente incluye una serie de poderes muy penetrantes de verificación y sanción, como el de inspección, de exigir la exhibición de libros y registros, de convocar asambleas, e incluso de sustituir a los gerentes de la cooperativa u ordenar su liquidación.

En principio, debo puntualizar que mis reflexiones de hoy se centrarán únicamente en el tema de la vigilancia cooperativa en sentido estricto, es decir, la actividad con la que se vigila el cumplimiento por parte de las cooperativas de las disposiciones legales y reglamentarias que definen y regulan su objetivo y estructura, es decir, su identidad de cooperativas, desde su constitución hasta su liquidación, o lo que es lo mismo, a lo largo de toda su existencia. El art. 35 de la ley Colombiana n. ${ }^{\circ} 454$ de 1998 me parece muy claro en este respecto cuando establece los objetivos y las finalidades de la gestión por parte de la Superintendencia ${ }^{34}$. Por lo tanto, no hablaré aquí de la auditoría o control de cuentas, porque esta actividad se ve influenciada muy limitadamente por la naturaleza cooperativa de la entidad que ha de ser controlada $y$, en cualquier caso, se refiere a todas las entidades y no sólo específicamente a las cooperativas ${ }^{35}$.

Además, me centraré particularmente en la supervisión de carácter general, es decir, referida a toda clase de cooperativas, y no examinaré aquella a la que están sujetas algunos tipos de cooperativas como las

34 Según el art. 35 de la ley 454 de 1998, por la cual se determina el marco conceptual que regula la economía solidaria, «La Superintendencia de la Economía Solidaria, en su carácter de autoridad técnica de supervisión desarrollará su gestión con los siguientes objetivos y finalidades generales: 1. Ejercer el control, inspección y vigilancia sobre las entidades que cobija su acción para asegurar el cumplimiento de las disposiciones legales y reglamentarias y de las normas contenidas en sus propios estatutos. 2. Proteger los intereses de los asociados de las organizaciones de Economía Solidaria, de los terceros y de la comunidad en general. 3. Velar por la preservación de la naturaleza jurídica de las entidades sometidas a su supervisión, en orden a hacer prevalecer sus valores, principios y características esenciales. 4. Vigilar la correcta aplicación de los recursos de estas entidades, así como la debida utilización de las ventajas normativas a ellas otorgadas. 5. Supervisar el cumplimiento del propósito socioeconómico no lucrativo que ha de guiar la organización y funcionamiento de las entidades vigiladas».

35 Sobre este tema, cf. recientemente A. ZUBIAURRE GURRUCHAGA, «La auditoría en las cooperativas españolas y francesas. Estudio comparativo de su regimen legal», en CIRIEC-España. Revista Jurídica de Economía Social y Cooperativa, número 25, 2014, p. 195 y ss. 
de ahorro y crédito o de seguro. Asimismo, no abordaré la particular supervisión a la que las cooperativas, como cualquier otra entidad incluso no cooperativa, están sujetas por razón de su objeto social específico como, por ejemplo, el de prestar determinados servicios públicos. Tampoco en este caso existe nada específico para las cooperativas.

Finalmente, aún a modo de premisa, mis reflexiones sobre la supervisión cooperativa pueden aplicarse también a la supervisión de las entidades de la economía solidaria en general.

La primera observación de carácter general concierne a la función de la supervisión cooperativa, y es que la supervisión cooperativa desempeña un papel fundamental para la defensa y promoción de esta forma jurídica de empresa y, por lo tanto, constituye un elemento esencial de la legislación cooperativa.

La razón fundamental por la que la supervisión constituye un factor de desarrollo de las cooperativas (así como de otras entidades de la economía solidaria) reside en el hecho de que la supervisión garantiza que la denominación de cooperativa no sea empleada por entidades cuyas finalidad y estructura no sean las de una cooperativa, es decir, que no tengan naturaleza cooperativa. De esta manera, la supervisión asegura que no se abuse del nombre de cooperativa para apropiarse de ventajas que derivan de la forma cooperativa a las que no se tenga derecho. Estas ventajas no son sólo las que el Estado podría proveer a las cooperativas, sino también las que son consecuencia del propio uso de la denominación de cooperativa, puesto que esta denominación (en razón de los valores y principios que lleva consigo) ejerce una fuerte atracción sobre terceros como son los consumidores, trabajadores, financiadores, etc., y de la pertenencia de cada cooperativa individual a un sistema cooperativo, a un movimiento cooperativo, que de por sí es fuente de ventajas.

Por lo tanto, la supervisión es la natural consecuencia de la identidad peculiar de las cooperativas. A través de la supervisión se preserva la naturaleza jurídica de la cooperativa, o dicho de otro modo, se protege la denominación de cooperativa, como si fuera una marca colectiva de calidad. Y, de este modo, se protegen varios intereses: el interés de los socios de la cooperativa en participar en entidades realmente cooperativas; el interés del Estado en fomentar la verdadera cooperación; el interés de terceros en relacionarse con verdaderas cooperativas; y por último, si bien no menos importante, se tutela el interés de todas las cooperativas y del movimiento cooperativo en general por beneficiarse y seguir beneficiándose de esta marca.

La supervisión, por lo tanto, no se relaciona solamente con el fomento estatal, cualquiera que sea su forma, una tributación especí- 
fica u otra, sino que satisface diversos intereses de variada naturaleza y que pertenecen a diferentes sujetos. En particular, la protección del interés de todas las cooperativas y del movimiento cooperativo en beneficiarse de la marca de cooperativa es un aspecto realmente fundamental. Muestra la estrecha conexión existente entre el tema de la supervisión y el de la cooperación entre cooperativas, que es central no sólo para justificar la supervisión como instituto del derecho cooperativo, sino también, como veremos, para discutir sobre sus modalidades concretas. El hecho es que la denominación de cooperativa es una marca colectiva, que, en cuanto tal, se valora y se devalúa en función de comportamientos conformes o no conformes a las reglas de su uso por parte de todos los que usan la marca, incluso un solo usuario. Esta marca, siendo colectiva, se expone al riesgo común a todo bien colectivo, el riesgo del comportamiento oportunista de quien quiera aprovecharse de las utilidades que el bien común genera sin soportar los costes asociados al uso del bien común (costes que, en el caso de la marca cooperativa, son el respeto de las reglas que forman la identidad cooperativa). Por lo tanto, se necesitan reglas que protejan el bien común frente a comportamientos oportunistas de este tipo que puedan conducir a la destrucción del bien común (mi referencia aquí es a la «tragedia de los bienes comunes» enunciada por Garrett Hardin) ${ }^{36}$. Y por lo que se refiere a la marca colectiva de cooperativa, estas reglas son precisamente las que prevén la supervisión cooperativa.

En conclusión, dada su función, la supervisión cooperativa es un instituto irrenunciable para la defensa y el desarrollo de las cooperativas, así como de todas las entidades de la economía solidaria a las que se presentan problemas del mismo tipo. No sorprende entonces que en el ordenamiento italiano, entre otros (incluida Colombia), incluso en la Constitución se haga referencia al control de las cooperativas $^{37}$. Por lo tanto, la cuestión real en el tema de la supervisión no es el "si» de la supervisión, sino el "cómo», es decir, qué formas de supervisión son más eficaces, eficientes y, sobre todo, respetuosas con la identidad cooperativa. Teniendo en cuenta este interrogante, vamos a ver qué modelos de supervisión nos ofrece el análisis comparativo de las leyes cooperativas existentes, para analizarlos a continuación de forma crítica.

36 Cf. G. HARDIN, «The Tragedy of the Commons», en 162 Science, n. 3859 , 1968, p. 1243 y ss.

37 Véase el art. 45 de la Constitución italiana (específicamente dedicado a las sociedades cooperativas) y el art. 189 , numeral 24 , de la Constitución colombiana (que, sin embargo, no abarca solamente las cooperativas). 
Sin contar los ordenamientos en los que la ley cooperativa no se ocupa en absoluto de la cuestión de la supervisión que, sin embargo, no son pocos (Finlandia, Holanda, México y Perú entre ellos), es posible identificar cuatro modelos legislativos distintos de supervisión.

1) En el primer modelo (que es el más difundido), el Estado se halla en posición central, en el sentido de que no sólo regula el fenómeno de la supervisión, sino también la ejerce directamente mediante un ente especial ubicado en el área de un ministerio, o en la oficina de la presidencia, o en otro lugar. Aunque con diferente intensidad, este modelo se encuentra en diversos ordenamientos y no sólo de América Latina (incluida Colombia). Se encuentra también en países con una economía liberal y desarrollada y, sobre todo, con un movimiento cooperativo consolidado, como el del Reino Unido (o Japón), donde hay una entidad pública que controla que las cooperativas sean genuinas o "de buena fe», como lo expresa la ley inglesa ${ }^{38}$.

2) El segundo modelo es completamente opuesto al primero. Lo ofrece, entre otras que se inspiran en ella, la ley alemana de cooperativas. Aquí, el Estado solamente regula el fenómeno de la supervisión, previendo que ésta sea ejercida por las propias cooperativas, o más bien por entidades de supervisión integradas por cooperativas de las que éstas deben ser afiliadas, siendo la afiliación a una entidad de supervisión una conditio sine qua non para la constitución y existencia de una cooperativa. Sin embargo, las entidades cooperativas de supervisión están sujetas al control del Estado. Cabe subrayar que las mismas entidades cooperativas pueden desarrollar una actividad de apoyo y promoción de las cooperativas afiliadas, pero son distintas de las cooperativas de segundo grado que las cooperativas pueden establecer para el desarrollo de una actividad económica en común 39 .

Entre estos dos extremos hay algunos modelos intermedios que combinan la presencia pública y la privada en el desempeño de la supervisión.

3) Un modelo intermedio es aquel en el que la supervisión la ejerce un ente especial instituido por la ley cooperativa, pero integrado también, o incluso en mayoría, por representantes del movimiento cooperativo. Para dar sólo dos ejemplos desde continentes diversos, este modelo se encuentra en Argentina y en Bélgica (y además en Brasil y Portugal). En Argentina, el «Instituto Nacional de Asociativismo y Economía Social» (INAES) está dirigido y administrado por un directorio

38 Cf. I. SNAITH, "United Kingdom», en D. CRACOGNA, A. FICl, H. HENRY (eds.), International Handbook of Cooperative Law, cit., p. 735 y ss.

39 Cf. H.-H. MÜNKNER, «Germany», cit. 
formado por un presidente y seis vocales, dos en representación del Poder Ejecutivo Nacional y cuatro en representación de las Asociaciones Mutuales y de las Cooperativas ${ }^{40}$. En Bélgica, el Consejo Nacional de la Cooperación forma parte del Ministerio de Asuntos Económicos, pero está compuesto por representantes del movimiento cooperativo, designados por grupos nacionales de cooperativas, así como por otras cooperativas no afiliadas a ellos ${ }^{41}$. Cabe subrayar que las mismas entidades están encargadas por ley también, entre otras, de la función de promoción de la cooperación.

4) Otro modelo intermedio es el que se encuentra en el ordenamiento jurídico italiano. Aquí la ley prevé que asociaciones nacionales de cooperativas, reconocidas por el Estado como organismos de supervisión, ejerzan la supervisión de las cooperativas afiliadas, mientras que el Estado ejerce la supervisión de las cooperativas no afiliadas a ninguna asociación, además de las asociaciones de cooperativas reconocidas como organismos de supervisión. En todo caso, el Estado puede someter a inspección extraordinaria a toda cooperativa, afiliada y no afiliada, cuando tenga un temor fundado de irregularidades. Cabe subrayar, en primer lugar, que, como ya hemos visto con respecto al modelo alemán, las antedichas asociaciones difieren de las cooperativas de segundo grado que las cooperativas italianas pueden constituir para desarrollar una actividad económica en común; y en segundo lugar, que a la hora de ejercer la supervisión de cooperativas no afiliadas, la ley permite al Estado servirse de supervisores procedentes de las asociaciones $^{42}$.

Si éstos son los modelos que surgen del análisis comparativo, sin embargo no se puede concluir en absoluto que uno sea mejor que el otro o preferible. De hecho, cada uno presenta posibles ventajas y desventajas y su efectividad depende mucho del contexto en el que ha de aplicarse. No obstante, es posible realizar algunos comentarios generales y abstractos como orientación para la elección o reforma del régimen existente. Debo subrayar además que estas reflexiones valen también con referencia a la supervisión de otras entidades de la economía social o solidaria, también considerando que hay una tendencia legisla-

40 Cf. D. CRACOGNA, «Argentina», en D. CRACOGNA, A. FICl, H. HENRY (eds.), International Handbook of Cooperative Law, cit., p. 189 y ss.

${ }^{41}$ Cf. A. COATES, «Belgium», en D. CRACOGNA, A. FICl, H. HENRY (eds.), International Handbook of Cooperative Law, cit., p. 251 y ss.

42 Véase A. FICl, «Italy», en D. CRACOGNA, A. FICl, H. HENRY (eds.), International Handbook of Cooperative Law, cit., p. 479 y ss.; A. FICI, «La cooperación entre cooperativas en el derecho italiano y comparado», en Boletín de la Asociación Internacional de Derecho Cooperativo, n. 48, 2014, p. 103 y ss. 
tiva a incluir el sector cooperativo en el sector de la economía social, y en particular, como demuestra el caso de Portugal (y como ocurrió en Argentina y en Colombia), a trasformar la entidad de supervisión cooperativa en una entidad de supervisión de la economía social ${ }^{43}$. En definitiva:

i) Cualquiera que sea el modelo de supervisión empleado, debe garantizarse la autonomía de las cooperativas tanto frente al Estado como frente a otras entidades (incluidas las federaciones de cooperativas) que la ejerzan en su lugar. Tanto la $\mathrm{ACl}$ como la OIT hacen hincapié en la necesidad de garantizar la autonomía e independencia de las cooperativas. En particular, la OIT invita a «prever la adopción de medidas de supervisión de las cooperativas acordes con su naturaleza y funciones, que respeten su autonomía y sean conformes con la legislación y la práctica nacionales y no menos favorables que las medidas aplicables a otras formas de empresa o de organización social» ${ }^{44}$.

ii) Para que la autonomía de las cooperativas sea garantizada, es necesario que, cuando el Estado sea el encargado del control, dicho control sea desempeñado por un organismo público diferente al encargado de la promoción cooperativa (como sucede en Colombia y no ocurre en Chile, por ejemplo)45; en cambio, cuando una entidad del movimiento cooperativo sea la encargada de la supervisión, es necesario que tal entidad no desarrolle también una actividad empresarial, sino que sólo se ocupe del control y, como mucho, de la promoción cooperativa.

iii) Para que la autonomía de las cooperativas sea garantizada, es necesario que la supervisión apunte hacia la legitimidad de la acción de la cooperativa (al respecto de las normas de ley o de reglamentos) y no hacia el mérito o la oportunidad de las decisiones empresariales.

iv) Además, para que la autonomía de las cooperativas sea garantizada, es necesario que las cooperativas puedan interponer recursos de carácter administrativo o judicial contra las resolucio-

43 En Portugal esta entitad es la «Cooperativa António Sérgio para a economia social» (CASES). En Argentina, el «Instituto Nacional de Asociativismo y Economía Social» (INAES). En Colombia, la "Superintendencia de la economía solidaria» (Supersolidaria).

44 Recomendación n. ${ }^{\circ}$ 193/2002 de 20 de junio de 2002, párrafo 6, c).

45 Cf. A.J. SARMIENTO REYES, «Colombia», en D. CRACOGNA, A. FICI, H. HENRY (eds.), International Handbook of Cooperative Law, cit., p. 355 y ss.; J. ALCALDE SILVA, «Chile», en D. CRACOGNA, A. FICI, H. HENRY (eds.), International Handbook of Cooperative Law, cit., p. 317 y ss. 
nes de la autoridad (pública o privada) encargada de la revisión, como ya prevé, por ejemplo, la Ley Marco para las cooperativas de América Latina ${ }^{46}$.

v) Los modelos intermedios que he presentado anteriormente son muy interesantes en la medida en que, por un lado, reducen, aunque sin anularlo, el papel público en beneficio del autogobierno o autocontrol cooperativo y, por otro, garantizan que la supervisión se desarrolle según lo que la ley prescribe, pudiendo el poder público controlar a los que controlan y así generar la suficiente confianza en la autenticidad del movimiento; además, estos modelos se alinean a la tendencia, señalada antes, a la privatización de funciones estatales relativas a cooperativas.

vi) En particular, el tercer modelo, el que prevé el organismo público integrado por representantes del movimiento cooperativo, parece un buen modelo cuando en un país no hay aún un movimiento cooperativo lo suficientemente consolidado, unido y desarrollado; mientras que el cuarto modelo, el italiano, presupone un movimiento cooperativo con estas características; a este último modelo hace referencia la Ley Marco para las cooperativas de América Latina cuando prevé la posibilidad de que la autoridad pública delegue la actividad de supervisión en las cooperativas de grado superior que ejerzan la representación del movimiento cooperativo 47 .

vii) Sin embargo, ambos modelos intermedios favorecen la unión entre cooperativas y la creación de un movimiento cooperativo sólido lo que, por lo tanto, vuelve a ser un valor añadido de estos modelos intermedios de regulación, si es verdad que la cooperación entre las cooperativas es un principio de identidad cooperativa de la $\mathrm{ACl}^{48}$, un objetivo autónomamente puesto por la OIT a cargo de los legisladores cooperativos y un factor de desarrollo de las cooperativas individuales ${ }^{49}$.

46 «Contra las resoluciones de la autoridad de aplicación que impongan sanciones podrán interponerse los recursos de carácter administrativo y judicial previstos por la legislación vigente» (art. 100).

47 «Por delegación de la autoridad de aplicación las cooperativas de grado superior que ejerzan representación del movimiento cooperativo podrán realizar actividades de supervisión. Asimismo podrán encargarse de actividades de registro por delegación de la autoridad encargada del Registro de Cooperativas» (art. 84, apartado 2).

48 El sexto principio, como sabemos.

49 Cf. A. FICl, «La cooperación entre cooperativas en el derecho italiano y comparado», cit. 
viii) Por último, cualquiera que sea el modelo de supervisión empleado, es necesario que se limiten los costes a cargo de las cooperativas, si es preciso, distinguiendo entre grandes y pequeñas cooperativas; es necesario que la supervisión no sea inútilmente invasiva; y que las personas que la ejerzan sean personas especialmente calificadas para ello.

\section{Bibliografía}

ALCALDE SILVA J., «Chile», en D. CRACOGNA, A. FICI, H. HENRY (eds.), International Handbook of Cooperative Law, Springer, Heidelberg, 2013.

COATES A., «Belgium», en D. CRACOGNA, A. FICl, H. HENRY (eds.), International Handbook of Cooperative Law, Springer, Heidelberg, 2013.

CRACOGNA D., "Problemas actuales del derecho cooperativo», en Revista de derecho privado y comunitario, número 3, 2011.

CRACOGNA D., "Argentina», en D. CRACOGNA, A. FICI, H. HENRY (eds.), International Handbook of Cooperative Law, Springer, Heidelberg, 2013.

CRACOGNA D., FICI, A., HENRY, H. (eds.), International Handbook of Cooperative Law, Springer, Heidelberg, 2013.

FAJARDO G., «La especificidad de las sociedades cooperativas frente a las sociedades mercantiles y la legitimidad de su particular régimen jurídico y fiscal según el tribunal de justicia de la Unión Europea», en Revista de derecho mercantil, 2013.

FAJARDO, G., FICl, A., HENRŸ, H., HIEZ, D., MEIRA, D., MÜNKNER, H. H., y SNAITH I., «El Nuevo grupo de estudio en Derecho cooperativo europeo y el proyecto "los principios del Derecho cooperativo europeo"», en CIRIECEspaña. Revista Jurídica de Economía Social y Cooperativa, número 24, 2013.

FAJARDO-CALDERÓN C.L., CABAL-CRUZ C.C., DONNEYS-BELTRÁN O.A., "La economía solidaria: de lo legal a la formación integral», en Criterio libre, número 9, 2008.

$\mathrm{FICl} \mathrm{A.,} \mathrm{"Cooperative} \mathrm{Identity} \mathrm{and} \mathrm{the} \mathrm{Law»,} \mathrm{en} \mathrm{European} \mathrm{Business} \mathrm{Law} \mathrm{Re-}$ view, 2013.

$\mathrm{FICl}$ A., "An introduction to cooperative law», en D. CRACOGNA, A. FICl, H. HENRY (eds.), International Handbook of Cooperative Law, Springer, Heidelberg, 2013.

FICI A., «Italy», en D. CRACOGNA, A. FICl, H. HENRY (eds.), International Handbook of Cooperative Law, Springer, Heidelberg, 2013.

$\mathrm{FICl}$ A., "La cooperación entre cooperativas en el derecho italiano y comparado», en Boletín de la Asociación Internacional de Derecho Cooperativo, n. 48, 2014.

$\mathrm{FICl}$ A., "La Sociedad Cooperativa Europea: cuestiones y perspectivas», en CIRIEC-España. Revista Jurídica de Economía Social y Cooperativa, número 25, 2014. 
$\mathrm{FICl}$ A., «The Essential Role of Cooperative Law», en Dovenschmidt Quarterly, 2014.

$\mathrm{FICl}$ A., «Función y modelos de regulación de la empresa social» (de próxima publicación).

HANSMANN H., The ownership of enterprise, HUP, Cambridge-London, 1996.

HARDIN G., "The Tragedy of the Commons», en 162 Science, n. ${ }^{\circ} 3859,1968$.

HENRŸ H., Orientaciones para la legislación cooperativa, Segunda edición, Organización Internacional del Trabajo, Ginebra, 2013.

HIEZ D. y TADJUDJE W., "The OHADA Cooperative Regulation», en D. CRACOGNA, A. $\mathrm{FICl}, \mathrm{H}$.

HENRY (EDS.), International Handbook of Cooperative Law, Springer, Heidelberg, 2013.

MÜNKNER H.-H., «Germany», en D. CRACOGNA, A. FICl, H. HENRY (eds.), International Handbook of Cooperative Law, Springer, Heidelberg, 2013.

SNAITH I., «United Kingdom», en D. CRACOGNA, A. FICl, H. HENRY (eds.), International Handbook of Cooperative Law, cit.

STIGLITZ J., "Moving beyond market fundamentalism to a more balanced economy», en Annals of Public and Cooperative Economy, 2009.

PETROU T., "Canada», en D. CRACOGNA, A. FICI, H. HENRY (eds.), International Handbook of Cooperative Law, Springer, Heidelberg, 2013.

SARMIENTO REYES A.J., "Colombia», en D. CRACOGNA, A. FICl, H. HENRY (eds.), International Handbook of Cooperative Law, Springer, Heidelberg, 2013.

ZUBIAURRE GURRUCHAGA A., "La auditoría en las cooperativas españolas y francesas. Estudio comparativo de su régimen legal», en CIRIEC-España. Revista Jurídica de Economía Social y Cooperativa, número 25, 2014. 